\title{
Vibrational Mean Amplitudes of Methylamine and Dimethylamine
}

\author{
Giovanna Dellepiane \\ Istituto di Chimica Industriale, Università di Genova \\ and C.N.R., Centro Nazionale di Chimica delle Macromolecole, Sez. V, Genova, Italy \\ and GiUseppe ZeRBI \\ Istituto di Chimica Industriale del Politecnico di Milano \\ and C.N.R., Centro Nazionale di Chimica delle Macromolecole, Sez. I, Milano, Italy \\ (Z. Naturforsch. 23a, 1661-1664 [1968]; received 26 April 1968)
}

\begin{abstract}
The mean amplitudes of vibration of methylamine and dimethylamine have been calculated from a previously reported Overlay Valence Force Field. Experimental and calculated $\mathrm{C}-\mathrm{N}$ mean amplitudes for methylamine are compared and discussed.
\end{abstract}

Mean amplitudes of vibration can be experimentally determined by electron diffraction studies of gases. They are known to be functions of the force constants $^{1}$ and therefore should provide additional information on molecular force fields. However, the large uncertainty of most vibrational amplitudes experimentally determined makes their applicability in the field just mentioned quite questionable ${ }^{2}$. For these reasons, when a "reliable", force field is known, it may be worth-while to derive from it spectroscopic mean amplitudes which can be used as a check on the force field when experimental amplitudes are available or as extra data to be incorporated into an electron diffraction analysis.

The purpose of this paper is to evaluate from an Overlay Valence Force Field previously reported ${ }^{3,4}$ the vibrational mean amplitudes of methylamine and dimethylamine. These data should be particularly interesting for methylamine, for which the results of an electron diffraction investigation are available $^{5}$.

\section{Geometry}

The molecular geometry adopted in the present work for methylamine and dimethylamine is shown in Figs. 1 und 2 respectively and explained in detail below.

\section{Methylamine}

Atoms 1, 4 and 5 of Fig. 1 lie in the $x z$ plane ( $x$ is positive for atoms $1,4,6$ and $7 ; z$ is positive

1 S. J. Cyvin, J. Mol. Spectry 3, 467 [1959]. - Y. Morino, Y. Nakamura, and T. IIJIMA, J. Chem. Phys. 32, 643 [1960].

2 S. J. Cyvin, B. N. Cyvin, J. Brunvoll, B. Andersen, and R. StøLEvik, Selected Topics in Structure Chemistry. Universitets-Forlaget, Oslo 1967.

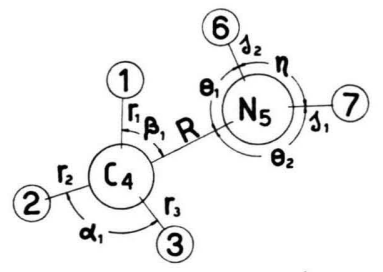

Fig. 1. Molecular geometry and internal coordinates of methylamine.

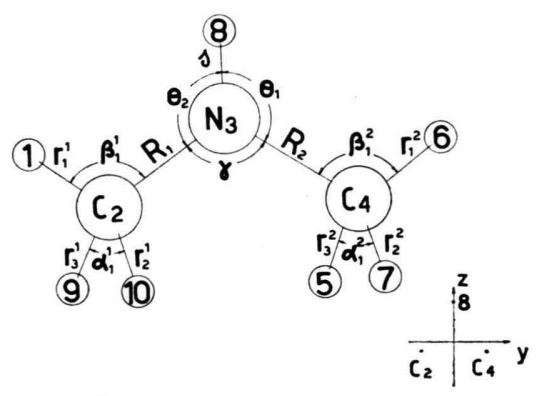

Fig. 2. Molecular geometry and internal coordinates of dimethylamine.

for atoms 1, 2, 3 and 4). The $y$ axis is pointing out of this symmetry plane with atoms 3 and 7 positive.

\section{Dimethylamine}

Atoms 3 and 8 of Fig. 2 lie in the plane of symmetry of the molecule. The $z$ axis lies in this plane $(z$ is positive for atoms $1,3,6$ and 8). The $y$ axis, perpendicular to the symmetry plane, is pointing towards atoms 4, 5, 6 and 7 . The $x$ axis has been taken in such a way that the coordinate system is left-handed.

3 G. Dellepiane and G. Zerbi, J. Chem. Phys., 48, 3573 [1968].

4 G. Dellepiane and G. Zerbi, Spectrochim. Acta, in press.

5 H. K. Higginbotham and L. S. Bartell, J. Chem. Phys. 42, 1131 [1965]. 


\section{Vibrational mean amplitudes}

The cartesian and internal mean square amplitude matrices have been calculated according to the equations 6

$$
\begin{aligned}
& \Sigma^{x}=T \Sigma^{Q} T^{\prime}, \\
& \Sigma^{R}=L \Sigma^{Q} L^{\prime}
\end{aligned}
$$

where $T$ and $L$ are the transformation matrices from cartesian and internal coordinates to normal coordinates and $\Sigma^{Q}$ is a diagonal matrix with the elements :

$$
\Sigma_{K}^{Q}=\frac{h}{8 \pi^{2} c v_{K}} \operatorname{coth}\left(\frac{h c v_{K}}{2 k T}\right) .
$$

Here $v_{K}$ is the vibrational frequency in $\mathrm{cm}^{-1}, h$ the Planck constant, $c$ the velocity of light, $k$ the Boltzmann constant, and $T$ the absolute temperature. The mean-square amplitude matrix for distances between non-bonded atoms is given by

$$
\Sigma^{q}=N \Sigma^{R} N^{\prime}
$$

where $N$ is the transformation matrix from non bonded distances to internal coordinates.

The mean amplitudes of vibration have been evaluated from the mean-square amplitude matrix elements ${ }^{7}$. The results obtained for the molecules studied in the present work are listed in Tables 1 to 4 .
A comparison between the experimental $\mathrm{C}-\mathrm{N}$ mean amplitudes of $\mathrm{CH}_{3} \mathrm{NH}_{2}$ and $\mathrm{CD}_{3} \mathrm{ND}_{2}$ and the calculated ones (see Table 2) shows that while the experimental $\mathrm{C}-\mathrm{N}$ amplitude sensibly changes from $\mathrm{CH}_{3} \mathrm{NH}_{2}$ to $\mathrm{CD}_{3} \mathrm{ND}_{2} \quad(u=0.0465 \pm 0.0015 \AA$ for $\mathrm{CH}_{3} \mathrm{NH}_{2} ; u=0.0512 \pm 0.0016 \AA$ for $\mathrm{CD}_{3} \mathrm{ND}_{2}$, Ref. ${ }^{5}$ ), the calculated values do not depend on the secondary isotope effects ${ }^{8}$. However, it should be noted that the large secondary isotope effect present in the experimental data should be considered

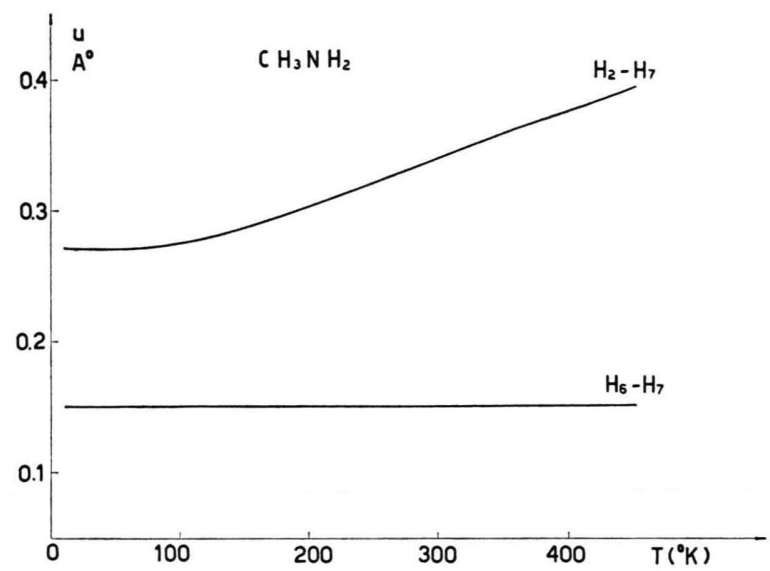

\begin{tabular}{|c|c|c|c|c|c|c|c|c|c|}
\hline \multirow{2}{*}{$\begin{array}{c}\text { Atom } \\
\text { number }\end{array}$} & \multirow{2}{*}{$\begin{array}{c}\begin{array}{c}\text { Cart- } \\
\text { esian } \\
\text { coordi- } \\
\text { nates }\end{array} \\
\end{array}$} & \multicolumn{2}{|c|}{$\mathrm{CH}_{3} \mathrm{NH}_{2}$} & \multicolumn{2}{|c|}{$\mathrm{CD}_{3} \mathrm{NH}_{2}$} & \multicolumn{2}{|c|}{$\mathrm{CH}_{3} \mathrm{ND}_{2}$} & \multicolumn{2}{|c|}{$\mathrm{CD}_{3} \mathrm{ND}_{2}$} \\
\hline & & $T=10$ & $\begin{array}{l}T=298.16 \\
\mathrm{C}\end{array}$ & $T=10$ & $r=298.16$ & $T=10$ & $T=298.16$ & $T=10$ & $r=298.16$ \\
\hline 1 & $\left\{\begin{array}{l}x \\
y \\
z\end{array}\right.$ & $\begin{array}{l}0.0757 \\
0.1194 \\
0.1090\end{array}$ & $\begin{array}{l}0.0757 \\
0.1241 \\
0.1090\end{array}$ & $\begin{array}{l}0.0620 \\
0.0824 \\
0.0868\end{array}$ & $\begin{array}{l}0.0620 \\
0.0854 \\
0.0868\end{array}$ & $\begin{array}{l}0.0755 \\
0.1408 \\
0.1112\end{array}$ & $\begin{array}{l}0.0756 \\
0.1522 \\
0.1112\end{array}$ & $\begin{array}{l}0.0620 \\
0.0967 \\
0.0889\end{array}$ & $\begin{array}{l}0.0620 \\
0.1057 \\
0.0890\end{array}$ \\
\hline 2,3 & $\left\{\begin{array}{l}x \\
y \\
z\end{array}\right.$ & $\begin{array}{l}0.1093 \\
0.0906 \\
0.1078\end{array}$ & $\begin{array}{l}0.1129 \\
0.0923 \\
0.1078\end{array}$ & $\begin{array}{l}0.0770 \\
0.0687 \\
0.0860\end{array}$ & $\begin{array}{l}0.0791 \\
0.0698 \\
0.0861\end{array}$ & $\begin{array}{l}0.1266 \\
0.0988 \\
0.1091\end{array}$ & $\begin{array}{l}0.1356 \\
0.1032 \\
0.1094\end{array}$ & $\begin{array}{l}0.0882 \\
0.0740 \\
0.0875\end{array}$ & $\begin{array}{l}0.0950 \\
0.0774 \\
0.0879\end{array}$ \\
\hline 4 & $\left\{\begin{array}{l}x \\
y \\
z\end{array}\right.$ & $\begin{array}{l}0.0173 \\
0.0175 \\
0.0273\end{array}$ & $\begin{array}{l}0.0173 \\
0.0175 \\
0.0273\end{array}$ & $\begin{array}{l}0.0229 \\
0.0229 \\
0.0278\end{array}$ & $\begin{array}{l}0.0229 \\
0.0229 \\
0.0278\end{array}$ & $\begin{array}{l}0.0175 \\
0.0183 \\
0.0284\end{array}$ & $\begin{array}{l}0.0175 \\
0.0183 \\
0.0284\end{array}$ & $\begin{array}{l}0.0230 \\
0.0231 \\
0.0287\end{array}$ & $\begin{array}{l}0.0230 \\
0.0231 \\
0.0287\end{array}$ \\
\hline 5 & $\left\{\begin{array}{l}x \\
y \\
z\end{array}\right.$ & $\begin{array}{l}0.0161 \\
0.0156 \\
0.0242\end{array}$ & $\begin{array}{l}0.0161 \\
0.0161 \\
0.0242\end{array}$ & $\begin{array}{l}0.0171 \\
0.0175 \\
0.0257\end{array}$ & $\begin{array}{l}0.0171 \\
0.0182 \\
0.0257\end{array}$ & $\begin{array}{l}0.0226 \\
0.0198 \\
0.0245\end{array}$ & $\begin{array}{l}0.0226 \\
0.0206 \\
0.0245\end{array}$ & $\begin{array}{l}0.0227 \\
0.0217 \\
0.0257\end{array}$ & $\begin{array}{l}0.0228 \\
0.0232 \\
0.0257\end{array}$ \\
\hline 6,7 & $\left\{\begin{array}{l}x \\
y \\
z\end{array}\right.$ & $\begin{array}{l}0.1504 \\
0.0963 \\
0.1145\end{array}$ & $\begin{array}{l}0.1586 \\
0.0994 \\
0.1145\end{array}$ & $\begin{array}{l}0.1672 \\
0.1033 \\
0.1159\end{array}$ & $\begin{array}{l}0.1797 \\
0.1083 \\
0.1160\end{array}$ & $\begin{array}{l}0.1064 \\
0.0705 \\
0.0907\end{array}$ & $\begin{array}{l}0.1154 \\
0.0729 \\
0.0908\end{array}$ & $\begin{array}{l}0.1247 \\
0.0762 \\
0.0924\end{array}$ & $\begin{array}{l}0.1408 \\
0.0811 \\
0.0926\end{array}$ \\
\hline
\end{tabular}

Fig. 3. Mean amplitudes of vibration $(u)$ for the $\mathrm{H}_{6} \cdots \mathrm{H}_{7}$ and $\mathrm{H}_{2} \cdots \mathrm{H}_{7}$ non-bonded distances of methylamine as functions of temperature.

Table 1. Cartesian mean amplitudes ( $\AA$ units) for methylamines from a 23 parameters Valence Force Field.

6 S. J. Cyvin, Acta Chem. Scand. 13, 2135 [1959]; see also J. H. SchachtschneIDER, Shell Development Company, Technical Report no. 57-65.
7 S. J. Cyvin, Spectrochim. Acta 15, 820, 835, 958 [1959]. 8 L. S. Bartell, J. Chem. Phys. 42, 1681 [1965]. 


\begin{tabular}{|c|c|c|c|c|c|c|c|c|c|c|c|c|}
\hline \multirow[b]{2}{*}{$\begin{array}{l}\text { Atom } \\
\text { pair }\end{array}$} & \multicolumn{3}{|c|}{$\mathrm{CH}_{3} \mathrm{NH}_{2}$} & \multicolumn{3}{|c|}{$\mathrm{CD}_{3} \mathrm{NH}_{2}$} & \multicolumn{3}{|c|}{$\mathrm{CH}_{3} \mathrm{ND}_{2}$} & \multicolumn{3}{|c|}{$\mathrm{CD}_{3} \mathrm{ND}_{2}$} \\
\hline & $T=10$ & $\begin{array}{c}273.16 \\
{\left[{ }^{\circ} \mathrm{K}\right]}\end{array}$ & 298.16 & $T=10$ & $\begin{array}{c}273.16 \\
{\left[{ }^{\circ} \mathrm{K}\right]}\end{array}$ & 298.16 & $T=10$ & $\begin{array}{c}273.16 \\
{\left[{ }^{\circ} \mathrm{K}\right]}\end{array}$ & 298.16 & $T=10$ & $\begin{array}{c}273.16 \\
{\left[{ }^{\circ} \mathrm{K}\right]}\end{array}$ & 298.16 \\
\hline $\mathrm{C}-\mathrm{H}$ & 0.0789 & 0.0789 & 0.0789 & .0675 & 0.0675 & 0.0675 & 0.0789 & 0.078 & 0.0789 & 0.0675 & 0.0675 & 0.0675 \\
\hline $\mathrm{C}-\mathrm{N}$ & 0487 & 0.0488 & 0.0489 & 0486 & 0.0488 & 0.0489 & 0.0486 & 0.0488 & 0.0489 & 0.0485 & 0.0487 & 0.0488 \\
\hline $\mathrm{N}-\mathrm{H}$ & 0.0725 & 0.0725 & 0.0725 & 0.0725 & 0.0725 & 0.0725 & 0.0620 & 0.0620 & 0.0620 & 0.0620 & 0.0620 & 0.0620 \\
\hline $\mathrm{H}_{2} \cdots \mathrm{H}_{3}$ & 0.1472 & 0.1473 & 0.1474 & 0.1266 & 0.1271 & 0.1274 & 0.1472 & 0.1473 & 0.1474 & 0.1266 & 0.1271 & 0.1274 \\
\hline $\mathrm{H}_{1} \cdots \mathrm{H}_{2}$ & 0.1476 & 0.1477 & 0.1478 & 0.1269 & 0.1276 & 0.1279 & 0.1476 & 0.1477 & 0.1478 & 0.1269 & 0.1276 & 0.1279 \\
\hline $\mathrm{H}_{1} \cdots \mathrm{N}$ & 0.1157 & 0.1159 & 0.1161 & 0.1010 & 0.1018 & 0.1022 & 0.1157 & 0.1159 & 0.1161 & 0.1009 & 0.1018 & 0.1022 \\
\hline $\mathrm{H}_{6} \cdots \mathrm{C}$ & 0.1350 & 0.1360 & 0.1365 & 0.1349 & 0.1360 & 0.1364 & 0.1174 & 0.1201 & 0.1211 & 0.1172 & 0.1200 & 0.1210 \\
\hline $\mathrm{H}_{6} \cdots \mathrm{H}_{7}$ & 0.1506 & 0.1508 & 0.1508 & 0.1506 & 0.1508 & 0.1508 & 0.1291 & 0.1297 & 0.1300 & 0.1291 & 0.1297 & 0.1300 \\
\hline $\mathrm{H}_{2} \cdots \mathrm{H}_{7}$ & 0.2722 & 0.3310 & 0.3403 & 0.2584 & 0.3243 & 0.3341 & 0.2490 & 0.3215 & 0.3316 & 0.2312 & 0.3145 & 0.3252 \\
\hline
\end{tabular}

Table 2. Mean amplitudes ( $\AA$ units) for methylamines from a 23 parameters Valence Force Field.

\begin{tabular}{|c|c|c|c|c|c|}
\hline \multirow{2}{*}{$\begin{array}{c}\begin{array}{c}\text { Atom } \\
\text { number }\end{array} \\
1,6\end{array}$} & \multirow{2}{*}{$\begin{array}{c}\begin{array}{c}\text { Cartesian } \\
\text { coordinates }\end{array} \\
\left\{\begin{array}{l}x \\
y \\
z\end{array}\right.\end{array}$} & \multicolumn{2}{|c|}{$\begin{array}{c}\mathrm{CH}_{3} \mathrm{NH} \mathrm{CH}_{3} \\
{\left[{ }^{\circ} \mathrm{K}\right]}\end{array}=298.16$} & \multicolumn{2}{|c|}{$\begin{array}{c}\mathrm{CH}_{3} \mathrm{ND} \mathrm{CH}_{3} \\
\quad\left[{ }^{\circ} \mathrm{K}\right]\end{array}=298.16$} \\
\hline & & $\begin{array}{l}0.1632 \\
0.0935 \\
0.1043\end{array}$ & $\begin{array}{l}0.1751 \\
0.0935 \\
0.1049\end{array}$ & $\begin{array}{l}0.1626 \\
0.0936 \\
0.1063\end{array}$ & $\begin{array}{l}0.1744 \\
0.0936 \\
0.1071\end{array}$ \\
\hline 2,4 & $\left\{\begin{array}{l}x \\
y \\
z\end{array}\right.$ & $\begin{array}{l}0.0229 \\
0.0364 \\
0.0235\end{array}$ & $\begin{array}{l}0.0240 \\
0.0371 \\
0.0237\end{array}$ & $\begin{array}{l}0.0232 \\
0.0364 \\
0.0238\end{array}$ & $\begin{array}{l}0.0244 \\
0.0372 \\
0.0239\end{array}$ \\
\hline 3 & $\left\{\begin{array}{l}x \\
y \\
z\end{array}\right.$ & $\begin{array}{l}0.0342 \\
0.0246 \\
0.0358\end{array}$ & $\begin{array}{l}0.0373 \\
0.0246 \\
0.0367\end{array}$ & $\begin{array}{l}0.0376 \\
0.0248 \\
0.0344\end{array}$ & $\begin{array}{l}0.0409 \\
0.0248 \\
0.0353\end{array}$ \\
\hline 5,9 & $\left\{\begin{array}{l}x \\
y \\
z\end{array}\right.$ & $\begin{array}{l}0.0957 \\
0.1444 \\
0.1367\end{array}$ & $\begin{array}{l}0.0990 \\
0.1521 \\
0.1444\end{array}$ & $\begin{array}{l}0.0904 \\
0.1444 \\
0.1423\end{array}$ & $\begin{array}{l}0.0929 \\
0.1521 \\
0.1503\end{array}$ \\
\hline 7,10 & $\left\{\begin{array}{l}x \\
y \\
z\end{array}\right.$ & $\begin{array}{l}0.1108 \\
0.1341 \\
0.1180\end{array}$ & $\begin{array}{l}0.1159 \\
0.1374 \\
0.1231\end{array}$ & $\begin{array}{l}0.1177 \\
0.1340 \\
0.1131\end{array}$ & $\begin{array}{l}0.1233 \\
0.1373 \\
0.1176\end{array}$ \\
\hline 8 & $\left\{\begin{array}{l}x \\
y \\
z\end{array}\right.$ & $\begin{array}{l}0.1117 \\
0.1076 \\
0.1208\end{array}$ & $\begin{array}{l}0.1126 \\
0.1076 \\
0.1216\end{array}$ & $\begin{array}{l}0.0892 \\
0.0869 \\
0.0927\end{array}$ & $\begin{array}{l}0.0906 \\
0.0869 \\
0.0939\end{array}$ \\
\hline
\end{tabular}

Table 3. Cartesian mean amplitudes ( $\AA$ units) for dimethylamines from a 23 parameters Valence Force Field.

\begin{tabular}{ccccccc}
\hline $\begin{array}{c}\text { Atom } \\
\text { pair }\end{array}$ & $T=10$ & $\begin{array}{c}\mathrm{CH}_{3} \mathrm{NH} \mathrm{CH}_{3} \\
273.16 \\
{\left[{ }^{\circ} \mathrm{K}\right]}\end{array}$ & 298.16 & $T=10$ & $\begin{array}{c}\mathrm{CH}_{3} \mathrm{ND} \mathrm{CH}_{3} \\
273.16 \\
{\left[{ }^{\circ} \mathrm{K}\right]}\end{array}$ & 298.16 \\
\hline $\mathrm{C}-\mathrm{H}$ & 0.0789 & 0.0789 & 0.0789 & 0.0789 & 0.0789 & 0.0789 \\
$\mathrm{C}-\mathrm{N}$ & 0.0488 & 0.0492 & 0.0493 & 0.0488 & 0.0492 & 0.0493 \\
$\mathrm{~N}-\mathrm{H}$ & 0.0732 & 0.0732 & 0.0732 & 0.0625 & 0.0625 & 0.0625 \\
$\mathrm{H}_{9} \cdots \mathrm{H}_{10}$ & 0.1475 & 0.1477 & 0.1478 & 0.1474 & 0.1477 & 0.1478 \\
$\mathrm{H}_{1} \cdots \mathrm{H}_{9}$ & 0.1474 & 0.1477 & 0.1478 & 0.1474 & 0.1477 & 0.1478 \\
$\mathrm{H}_{1} \cdots \mathrm{H}_{10}$ & 0.1466 & 0.1467 & 0.1468 & 0.1466 & 0.1467 & 0.1468 \\
$\mathrm{H}_{1} \cdots \mathrm{N}$ & 0.1158 & 0.1161 & 0.1162 & 0.1158 & 0.1161 & 0.1162 \\
$\mathrm{C}_{2} \cdots \mathrm{C}_{4}$ & 0.0719 & 0.0839 & 0.0860 & 0.0716 & 0.0838 & 0.0860 \\
$\mathrm{H}_{8} \cdots \mathrm{H}_{10}$ & 0.2275 & 0.2544 & 0.2593 & 0.2123 & 0.2437 & 0.2493 \\
$\mathrm{H}_{1} \cdots \mathrm{C}_{4}$ & 0.1844 & 0.2300 & 0.2367 & 0.1843 & 0.2300 & 0.2367 \\
$\mathrm{H}_{8} \cdots \mathrm{C}_{2}$ & 0.1334 & 0.1355 & 0.1408 & 0.1169 & 0.1254 & 0.1271 \\
\hline
\end{tabular}

Table 4. Mean amplitudes ( $\AA$ units) for dimethylamines from a 23 parameters_Valence Force Field. 
with caution as suggested by the authors themselves ${ }^{5}$. Our calculated value of $u=0.0488 \AA$ is an average value of the two reported $\mathrm{C}-\mathrm{N}$ mean amplitudes. No experimental amplitudes of vibration are so far available for dimethylamine.

The $\mathrm{C}-\mathrm{H}$ amplitudes of vibration reported in Tables 3 and 4 should be considered with reservation because the $\mathrm{C}-\mathrm{H}$ stretching vibrations are strongly affected by anharmonicity and no anhar- monic correction on the experimental frequencies has been made.

The influence of the temperature on the vibrational mean amplitude $(u)$ of methylamine has been examined over a small range of temperatures (10$450^{\circ} \mathrm{K}$ ). A plot of two $u$ values vs. temperature is reported in Fig. 3. A sensible temperature effect is observed only for the $\mathrm{H}_{2} \cdots \mathrm{H}_{7}$ non bonded distance. 